\title{
Chaque jour comporte son lot d'émotions
}

\section{Eva Mell}

Rédactrice en cheffe adjointe du Bulletin des médecins suisses

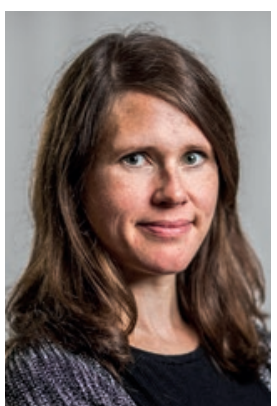

Dans notre famille, nous avons pour devise de vivre chaque émotion au moins une fois pleinement chaque jour. J’ai deux filles âgées de 2 et 5 ans. Les grandes joies côtoient les gros chagrins, sans compter les frustrations, vexations, minideuils et autres déceptions, mais aussi les plaisirs, la tendresse, la fierté et la satisfaction. En tant que parents, nous sommes au cœur de ce tourbillon émotionnel et veillons à ne pas nous laisser emporter d'une part et, de l'autre, à ce que nos enfants puissent vivre leurs émotions sans être submergées.

Et tout cela après une journée de travail parfois tout autant remplie en émotions. Parfois c'est le doute de soi qui domine, parfois la satisfaction d'avoir accompli le travail, et au final, le temps manque pour réfléchir aux nombreuses émotions traversées. Il peut aussi arriver qu'à la fin d'une journée de travail, tout le monde ait les nerfs à vif. Cette situation, on la connaît tous. Or, comment apprendre à gérer nos émotions et à les appréhender avec calme? Pour parvenir à gérer leur stress et leurs émotions, de nombreuses personnes se tournent vers les techniques de méditation bouddhistes venues d'Asie.

Grâce à cette technique de méditation, on peut observer ces émotions sous un autre angle afin de mieux comprendre pourquoi elles sont là.

Pour ma part, je n'ai pas besoin d'aller si loin. A Thoune, un psychiatre pour enfants et adolescents a développé une approche différente des émotions dites négatives pour gagner en sérénité. Sa méthode: la méditation sur les émotions basée sur l'hypnose (Hypnotische Gefühlsmeditation [HGM]) qui combine méditation, autohypnose et régulation des émotions. Elle est très simple à pratiquer. On se concentre en alternance d'abord sur l'émotion puis sur une image intérieure qui la symbolise. On porte donc son attention sur l'émotion, ensuite sur l'image, puis à nouveau sur l'émotion, puis retour sur l'image et ainsi de suite. Dans son livre Badlands-Meditationen [1] (non traduit en français), ce psychiatre, qui a été superviseur et formateur auprès de la Société médicale suisse d'hypnose (SMSH) jusqu'en 2021 et qui est désormais membre de l'association «hygs», décrit sa méthode: "L'émotion menaçante est mise à distance grâce à la focalisation et devient ainsi gérable» (trad. FMH). Résultat: frustration, colère ou désespoir ne m'atteignent plus de manière aussi forte. Il devient possible d'observer ces émotions sous différents angles de manière concentrée afin de mieux comprendre pourquoi elles sont là. Car il en va des émotions négatives comme des atomes, lit-on dans le livre. Ils ont une charge extérieure négative, mais un noyau positif. Exactement comme les émotions. La

\section{Chaque émotion négative a sa raison d'être.}

Se lier d'amitié avec elle est la tâche de toute une vie et peut se révéler éprouvante.

frustration me montre ce que j'aimerais atteindre, la tristesse ce qui m'est vraiment cher et important. Chaque émotion négative a sa raison d'être et peut mener à quelque chose de positif. Y réfléchir, découvrir la véritable nature de l'émotion, la catégoriser et, au final, se lier d'amitié avec elle, est la tâche de toute une vie et peut se révéler éprouvante. Je le vois aussi bien chez mes enfants que chez moi. Et dans les phases les plus difficiles, la méditation sur les émotions basée sur l'hypnose m'a permis de retrouver le calme.

L'auteur dispose d'ailleurs de sa propre chaîne YouTube où l'on peut expérimenter sa méthode au travers de méditations guidées et classées par émotions [2]. Comme toute chose dans la vie, cela nécessite un certain effort. Les vidéos durent 20 minutes en moyenne, temps qu'il faut pouvoir être au calme et disposé à essayer quelque chose de nouveau. Il est évident qu'il ne faut pas s'attendre à des miracles après un premier essai. Pour que la méthode déploie ses bienfaits, il faut de la discipline et de la persévérance, prévient le psychiatre. Mais là aussi, la patience est gage de succès. Chaque jour nous offre l'occasion de pratiquer, car qu'on le veuille ou non, les émotions surviennent au quotidien.

\section{Références}

1 www.hypnose-hgm.com/publikationen/ (en allemand seulement) 2 www.youtube.com/channel/UCoeHKFKmGPxuBuRkj-wMXwQ 